\title{
Influence of physical exercise on serum digoxin concentration and heart rate in patients with atrial fibrillation
}

\author{
Hans Erik Bøtker, Peter Toft, Niels Anders Klitgaard, Erik Efsen Simonsen
}

\begin{abstract}
Heart rate and serum digoxin concentration in eight patients with atrial fibrillation were studied at rest and during exercise when initial serum digoxin concentrations were zero and at low and high therapeutic values. Eight patients with ischaemic heart disease and in sinus rhythm were studied for comparison. Though the serum digoxin concentration decreased significantly during exercise, the absolute reduction in heart rate was the same at rest and during exercise in patients with atrial fibrillation. Compared with the control patients in sinus rhythm, the heart rate in patients with atrial fibrillation was not adequately controlled during exercise by any serum digoxin concentration tested despite a reduction in heart rate with increasing digoxin concentration. The effects of digoxin on heart rate regulation in atrial fibrillation are complex and include direct effects on the myocardium as well as indirect effects mediated by modulation of the autonomic nervous system; the present results indicate that the drug is not displaced from the target organs by decreasing serum concentrations during exercise.

In atrial fibrillation, because the demands on the filter function of the atrioventricular node are highly unphysiological, the effect of digoxin on heart rate during exercise is not adequate.
\end{abstract}

Digitalis is used to control the ventricular rate in patients with atrial fibrillation. Though it is effective at rest it may not prevent excessive increases in heart rate during exercise. ${ }^{1-3}$ One possible explanation for this is that the pharmacokinetics of digoxin change during exercise. Serum digoxin concentration decreased during exercise in several studies. ${ }^{4-6}$ This decrease has been shown to be related to an increased binding of digoxin to skeletal muscle during exercise. ${ }^{4}$ The mechanism underlying this finding probably involves an increased digoxin binding as a consequence of an increased $\mathrm{Na}-\mathrm{K}$ ATPase activity. ${ }^{7}$ The digoxin concentration in cardiac muscle in patients with a high heart rate caused by atrial fibrillation was higher than in patients with a lower heart rate in sinus rhythm. ${ }^{8}$ Because most of the digoxin is bound to skeletal muscle, a redistribution of digoxin during exercise might influence the amount of digoxin in other tissues. In fact a shift of digoxin from the erythrocytes to exercising skeletal muscle has been confirmed. ${ }^{5}$ Whether digoxin shifts from the target organs to the skeletal muscle during exercise is not known, and such a shift is not easy to measure directly in humans. We investigated the effect of digoxin on heart rate in patients with atrial fibrillation to see whether an exercise induced decrease in serum digoxin concentration was followed by an impaired heart rate reduction.

\section{Patients and methods}

We studied eight men (mean age $53 \cdot 3$ (27-61) years; weight $82 \cdot 1(67-101) \mathrm{kg}$ ) with atrial fibrillation. All had experienced atrial fibrillation for at least six months. The aetiology of atrial fibrillation was ischaemic heart disease in five, previous cardiac constriction in one, and lone atrial fibrillation in two cases. Patients with clinical or echocardiographic features of valvar heart disease were excluded. None had clinical or radiological evidence of congestive heart failure. None had angina pectoris. All were normotensive, had normal results of thyroid function tests, and normal serum electrolyte concentrations. None was taking medication other than digoxin.

For comparison we studied eight men (mean age $53 \cdot 3(39-62)$ years; weight $77 \cdot 1(65-90) \mathrm{kg}$ ) with ischaemic heart disease in sinus rhythm. The diagnosis of ischaemic heart disease was based upon previous myocardial infarction. None had clinical or radiological signs of congestive heart failure. None had angina pectoris. All were normotensive and had normal thyroid function tests and serum electrolyte concentrations. No medication was given for one week before the study.

Patients with atrial fibrillation performed three series of exercise tests at intervals of two weeks. The dosage of digoxin was varied in an attempt to obtain three different serum digoxin concentrations: zero and low and high within therapeutic range $(0 \cdot 6-2 \cdot 6 \mathrm{ng} / \mathrm{ml})$. Doses to be given to each patient were determined by reference to the patient's maintenance dose and serum concentration before the trial. To control for the development of tolerance to the actions of digoxin during the study ${ }^{9}$ the order of treatments was randomised. Dose schedules were kept constant for at least 10 days before each exercise test. Serum digoxin concentrations were analysed in triplicate with a commercial radioimmunoassay (Diagnostic Products). The coefficient of variation of the analysis was $2.8 \%$ at a mean value of $0.6 \mathrm{ng} / \mathrm{ml}$ and $2.0 \%$ at a mean value of $1.8 \mathrm{ng} / \mathrm{ml}$. 
Control patients in sinus rhythm performed one series of exercise tests.

On each study day the patient did three bicycle tests at increasing workloads $(25 \mathrm{~W}, 50$ W, and $75 \mathrm{~W}$ ). Each exercise test lasted 20 minutes and was followed by a one hour supine rest period. Blood samples were drawn and heart rates recorded every five minutes during the exercise periods and every 15 minutes during the resting periods. To take account of the considerable variation of heart rate in atrial fibrillation, heart rates were recorded over one minute at the end of each period.

Analysis of variance with repeated measures was used to compare changes in serum digoxin concentration at rest and during exercise. The changes in heart rate and blood pressure were analysed by two way analysis of variance, while the comparisons with the controls were evaluated by one way analysis of variance plus Dunnett's test. ${ }^{10}$ When $p<0.05$ the difference was regarded as significant.

The study was approved by the regional scientific ethics committee.

\section{Results}

SERUM DIGOXIN CONCENTRATIONS

Figure 1 shows the mean (SEM) serum digoxin concentration during rest and during exercise. The mean initial value was $0.87(0.10) \mathrm{ng} / \mathrm{ml}$ at

Figure 1 Serum digoxin concentration (mean (SEM)) (at high and low therapeutic

concentrations) at rest and during exercise.
Figure 2 Heart rates (mean (SEM)) at rest and during exercise in patients with atrial fibrillation at zero, low and high digoxin concentration. Heart rates from control patients in sinus rhythm are shown for comparison.
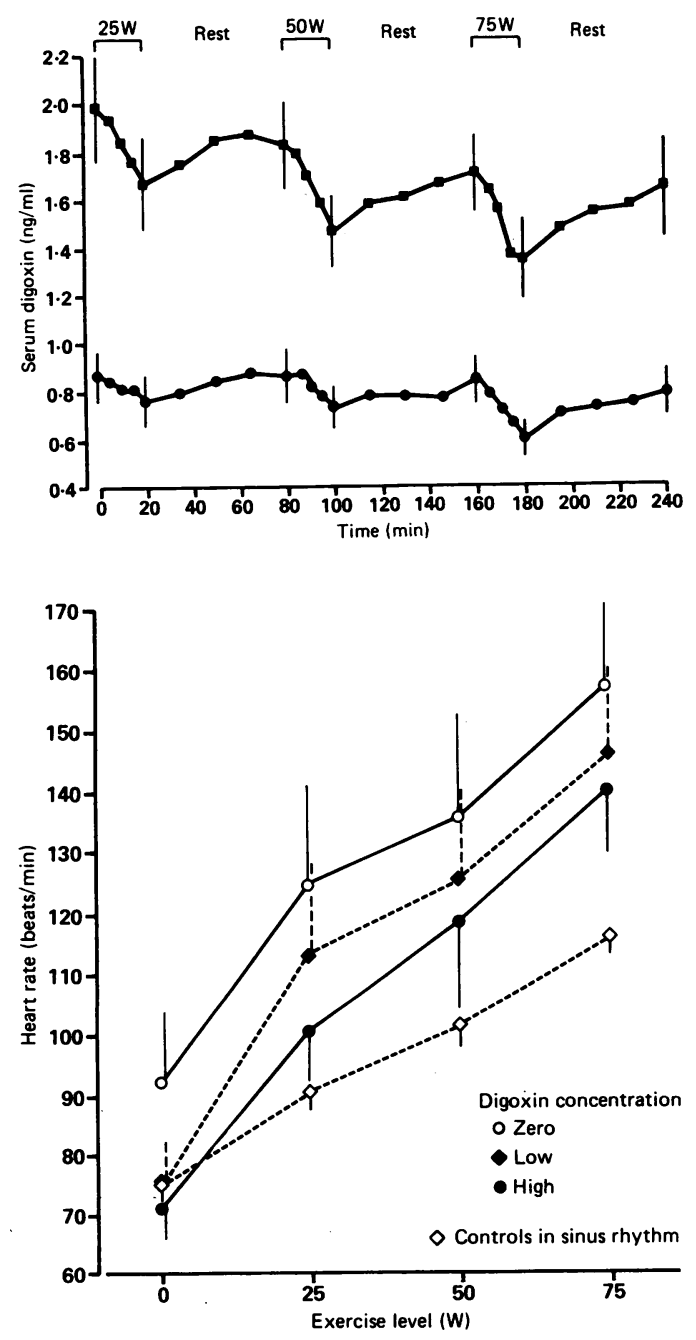

low digoxin doses and $1.98(0.22) \mathrm{ng} / \mathrm{ml}$ at high digoxin doses. In both groups serum digoxin concentration decreased significantly at each workload ( $\mathrm{p}<0.01)$ and returned almost to the initial value after an hour's rest. The decrease in serum digoxin concentration depended on workload $(p<0.05)$ and was directly proportional to the initial serum digoxin concentration $(\mathrm{r}=0.92, \mathrm{p}<0.01$ at low digoxin concentration and $r=0.91$, $\mathrm{p}<0.01$ at high digoxin concentration).

\section{HEART RATE DATA}

Figure 2 shows heart rates at rest and during exercise at zero, low, and high serum digoxin concentration in patients with atrial fibrillation compared with control patients in sinus rhythm. In patients with atrial fibrillation, ventricular rates at rest and at all levels of exercise decreased with each increment in digoxin concentration $(p<0.05)$, though no exact correlation between serum digoxin concentration and heart rate reduction was shown. As indicated by the parallel course of heart rate curves from patients with atrial fibrillation (fig 2) the absolute reduction in heart rate was similar at rest and during exercise. The patients with atrial fibrillation showed considerable variability in heart rate, but all patients retained their own characteristic rate pattern at various digoxin concentrations. Compared with the control group, however, only the heart rate at rest was sufficiently controlled whereas all levels of exercise caused a significant increase in heart rate $(p<0.05$ at $25 \mathrm{~W}$ and $50 \mathrm{~W}$, $\mathrm{p}<0.01$ at $75 \mathrm{~W}$ ), presumably because of a greater susceptibility to physical activity in atrial fibrillation. Figure 3 shows the increase in heart rates with increasing workload and the lack of association with serum digoxin concentration.

\section{LOG CONCENTRATION-RESPONSE RELATIONS}

Because the increase in heart rate and reduction in serum digoxin concentrations were both related to workload, log concentrationresponse relations were evaluated at each workload separately. We found no relation between

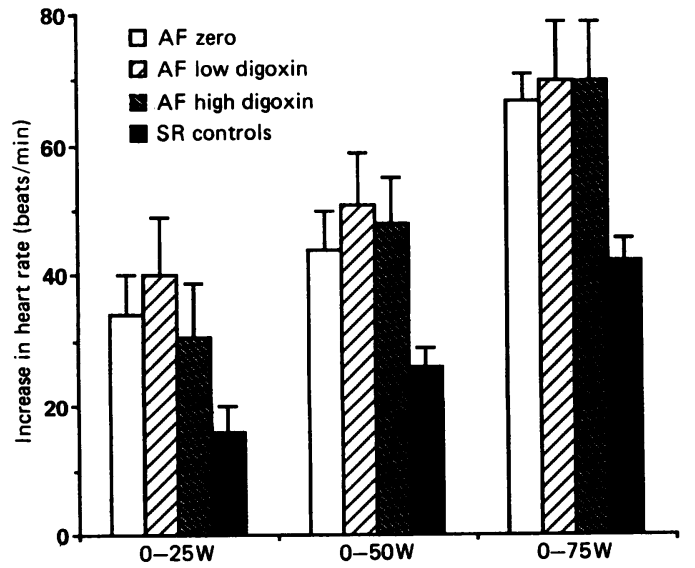

Figure 3 Increase in heart rate (mean (SEM)) from rest to $25 \mathrm{~W}, 50 \mathrm{~W}$, and $75 \mathrm{~W}$ respectively in controls with sinus rhythm and in patients with atrial fibrillation at zero, low, and high digoxin concentration. AF, atrial fibrillation; SR, sinus rhythm. 
Figure 4 Relation between increase in heart rate and decrease in serum digoxin concentration in patients with atrial fibrillation at $25 \mathrm{~W}, 50 \mathrm{~W}$, and $75 \mathrm{~W}$ exercise.
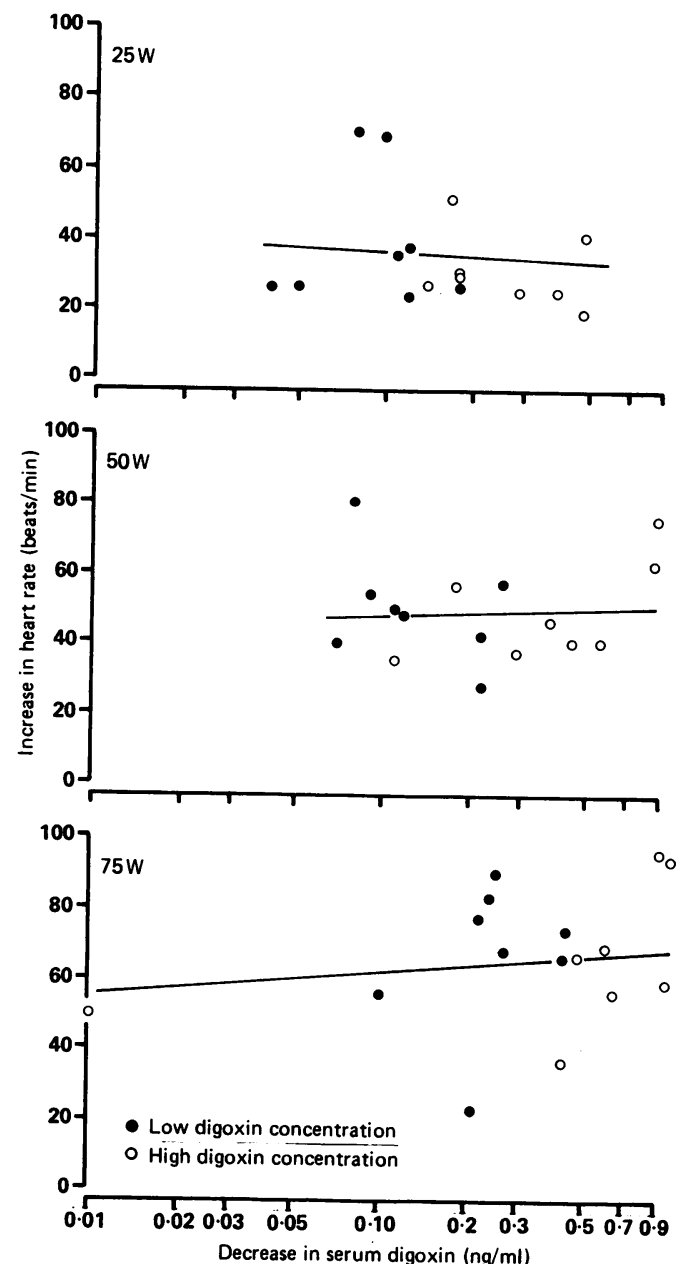

increase in heart rate and decrease in serum digoxin concentration $(\mathrm{r}=-0 \cdot 30$, NS at $25 \mathrm{~W}, \mathrm{r}=0.25, \mathrm{NS}$ at $50 \mathrm{~W}$, and $\mathrm{r}=0.35, \mathrm{NS}$ at $75 \mathrm{~W}$ ) (fig 4.).

HAEMODYNAMIC DATA

Changes in mean blood pressure during exercise were not affected by digoxin.

\section{Discussion}

The results of the present study confirm the findings of previous studies ${ }^{1-3}$ that digoxin was not able to abolish the excessive increases in heart rate during exercise in atrial fibrillation. Our results also show that the increase in ventricular rate was greater in atrial fibrillation than in matched controls in sinus rhythm. The capacity of digoxin to reduce heart rate, however, did not decrease during exercise in atrial fibrillation. Mean heart rate decreased as the digoxin concentration increased and the reduction in heart rate was of equal magnitude at rest and during exercise, indicating that the increase in heart rate with exercise is the same whether or not the patients are taking digoxin. Consequently, the inadequate control of exercise heart rate in digoxin treated atrial fibrillation is rather due to the disease per se than to altered pharmacokinetics during exercise.

ROLE OF THE ATRIOVENTRICULAR NODE IN ATRIAL FIBRILLATION

The way in which the atrioventricular node scales the rapid atrial activity to a slower ventricular response in atrial fibrillation is controversial. The classic electrophysiological mechanism used to explain atrioventricular transmission includes a conduction of atrial excitation through the atrioventricular node to the His-Purkinje system and the ventricular myocardium. In normal sinus rhythm the atrioventricular node acts only as a delaying structure. The ventricular rate is directly related to the effect of stress on the firing frequency of the sinoatrial node and is not affected by autonomically induced changes in the atrioventricular nodal conduction. In atrial fibrillation the atrioventricular node is constantly bombarded by $400-600$ impulses every minute. Thus atrioventricular nodal conductivity becomes the only regulating mechanism capable of controlling the ventricular rate, and the node has the characteristics of a filter. ${ }^{1}$ The ventricular rate in atrial fibrillation is the ultimate outcome of a complex interplay of several factors, which can be grouped into three major categories: (a) the nature of the electrical input into the atrioventricular node, that is, the number, irregularity, and direction of atrial impulses that bombard the approaches to the atrioventricular node; $(b)$ the responsiveness of the atrioventricular nodal cells and the preservation of intranodal conduction; and (c) the modulation of $(a)$ and $(b)$ by extracardiac factors such as sympathetic and parasympathetic influences and the effects of drugs. In stress or exercise, during which the sympathetic tone is increased and vagal tone decreased, atrioventricular nodal conduction is enhanced resulting in excessive impulse transmission and disproportionately high ventricular rates, far beyond the "normal" and expected acceleration produced by similar stress and exercise in sinus rhythm.

The action of the atrioventricular node as a pacemaker/oscillator electrotonically modulated by atrial activity ${ }^{11-13}$ and also influenced by the autonomic nervous system ${ }^{14} 15$ has recently been proposed. Conclusive evidence in support of one specific mechanism in atrioventricular transmission has yet to be presented. ${ }^{1617}$ Our study was not directed toward the solution of this problem and does not allow any conclusions on the mechanism(s). Our results could accord with both mechanisms. However, until the contribution of autonomic influence on atrioventricular nodal pacemaker activity in the excessive increase in ventricular rate during exercise has been clarified, the atrioventricular nodal conduction theory offers a satisfactory explanation of the atrioventricular transmission.

EFFECT OF DIGOXIN IN ATRIAL FIBRILLATION

Digoxin decreases the ventricular rate in atrial fibrillation through two synergistic pharmacological actions: $(a)$ increase in the refractory period of the atrioventricular node and $(b)$ decrease in the refractory period of the atrial myocardium. Both actions are mediated by direct effects on the myocardium and by indirect effects owing to a modification of both autonomic neural activity and the sensitivity of 
the heart to vagal and sympathetic neurotransmitters. ${ }^{1819} \mathrm{~A}$ decrease in the refractory period of the atrial myocardium results in more atrial impulses reaching the atrioventricular junction in a given unit of time. More atrial impulses result in a greater degree of concealed conduction $^{20}$ in the atrioventricular node and thus in a slower ventricular rate. The ventricular rhythm remains random during digitalis treatment. ${ }^{18}$

Our results show that digoxin is capable of exerting the same effect (evaluated as a reduction in heart rate in absolute terms) at rest and during exercise. At rest an adequate reduction in heart rate is achieved. During exercise, however, vagal tone decreases and sympathetic tone increases; this overrides the effects of digoxin.

\section{RELATION BETWEEN SERUM DIGOXIN CONCENTRATION AND VENTRICULAR RATE RESPONSE}

Several studies have shown a correlation between serum digoxin and the myocardial concentration of the drug. ${ }^{821} 22$ Further, the myocardial uptake is related to the beating frequency of the heart. ${ }^{82324}$ In keeping with this the atrial concentration of digoxin was higher in patients in atrial fibrillation than in patients in sinus rhythm. ${ }^{8}$ The relation between serum and atrial concentrations is linear in patients in sinus rhythm but not in those in atrial fibrillation. ${ }^{8}$ Because of the various effects of digoxin the use of serum digoxin concentration in clinical practice must be based on the assumption that it reflects the degree of digitalisation of the patient and not only the relation between serum and the myocardium. The correlation between serum digoxin concentration and heart rate response is not exact..$^{25}$ None the less, mean falls in heart rate with increasing digoxin concentration suggest that serum digoxin concentration reflects the concentration at target organs.

The results from the present study reflect the combined effects of digoxin and do not allow us to distinguish between the direct and indirect effects. The direct effect of digoxin may be unchanged because the tendency for the myocardial digoxin concentration to decrease is offset by a concomitant increase in myocardial binding of digoxin as a secondary effect of the increased heart rate. ${ }^{8}$

The indirect actions of digoxin are very complex and not well understood. ${ }^{19}$ These actions take place in the myocardium as well as in the central nervous system, the peripheral autonomic ganglia and nerves, the arterial baroreceptors, and in the carotid sinus nerves. Furthermore, several if not all of the mechanisms just mentioned are influenced by changes in the heart and circulation that modify input to the autonomic nervous system. We cannot rule out the influence of the decreased serum digoxin concentration during exercise on the indirect effects. If there is such an influence, several mechanisms may counteract the decreased indirect effect on heart rate reduction. It is impossible to unravel every aspect of the effect of digoxin on heart rate.

\section{CLINICAL IMPLICATIONS}

The results of the present study explain some of the difficulties associated with the use of serum digoxin as a guide for digoxin treatment and its failure to prevent toxic reactions in patients. ${ }^{27}$ Because serum digoxin is affected not only by exercise but also by everyday physical activity $^{62829}$ we recommend a standard rest period before blood sampling.

Our results indicate that the decrease in serum digoxin during exercise is of minor clinical importance in terms of heart rate regulation. The excessive increase in heart rate during exercise in atrial fibrillation reflects the nature of the disease.

Though an increased dose of digoxin would be of benefit even during exercise ${ }^{30}$ it would cause only a slight improvement and increase the risk of digitalis toxicity. ${ }^{31}$ To ensure the best possible effect and avoid reaching a toxic concentration the dose of digoxin is adjusted according to the heart rate response and serum concentration at rest. In patients with excessive increases in heart rate during exercise, digoxin alone cannot be expected to give an adequate reduction during exercise. Treatment with a calcium channel blocker ensures a better heart rate regulation both at rest and during exercise in patients with chronic atrial fibrillation. ${ }^{132} 33$ Unfortunately, the superior control of exercise heart rate achieved with these agents is not associated with improved exercise endurance or "well-being" scores. ${ }^{32}$ Further side effects are more common with calcium channel blockers in the doses needed than with digoxin or the combination of calcium channel blockers and digoxin..$^{32}$ Previous studies of the association between heart rate reduction and exercise capacity and symptomatic improvement during treatment with the combination of digoxin and either a calcium channel blocker or a $\beta$ blocker have shown conflicting results; it is still not known whether a further reduction of heart rate during exercise is of any benefit. ${ }^{32-39}$ When digoxin alone does not give an adequate response, we prefer the addition of a drug with an alternative mode of action such as a calcium channel blocker or a $\beta$ blocker so that there is no need to approach the threshold of toxicity. Digoxin is not ideal but it is still a mainstay in the treatment of sustained atrial fibrillation.

This study was supported by grants from the Medical Research Foundation of Ringkøbing, Ribe og Sønderjylland County Hospitals, and the Danish Medical Research Council.

1 Klein HO, Kaplinsky E. Verapamil and digoxin: their respective effects on atrial fibrillation and their interaction. respective effects on atrial fibrillatic

2 Goldman S, Probst P, Selzer A, Cohn K. Inefficacy of "therapeutic" serum levels of digoxin in controlling the ventricular rate in atrial fibrillation. $\mathrm{Am} J$ Cardio 1975;35:651-5

3 Beasly R, Smith DA, McHaffie DJ. Exercise heart rates a different serum digoxin concentrations in patients with atrial fibrillation. $B M J 1985 ; 290: 9-11$.

4 Pedersen KE, Madsen J, Kjær J, Klitgaard NA, Hvidt S. Effects of physical activity and immobilisation on plasma digoxin concentration and renal digoxin clearance. Clin Pharmacol Ther 1983;34:303-8.

5 Joreteg T, Jogestrand T. Physical exercise and binding of digoxin to skeletal muscle-effect of muscle activation frequency. Eur J Clin Pharmacol 1984;27:567-70.

6 Jogestrand T. Influence of everyday physical activity on the serum digoxin concentration in digoxin treated patients. Clin Physiol 1981;1:209-14.

7 Lüllmann H, Peter T, Ziegler A. Kinetic events determining 
the effects of cardiac glycosides. Trends Pharmacol Sci 1979;1:102-6.

8 Jogestrand T. Digoxin concentration in right atrial myocardium, skeletal muscle and serum in man: influence of atria rhythm. Eur J Clin Pharmacol 1980;17:243-50.

9 Ford AR, Aronson JK, Graham-Smith DG, Carver JG. The acute changes seen in cardiac glycoside receptor sites, 86rubidium uptake and intracellular sodium concentration in the erythrocytes of patients during the early phases of digoxin therapy are not found during chronic therapy: pharmacological and therapeutic implications for chronic digoxin therapy. Br J Clin Pharmacol 1979;8:135-42.

10 Wallenstein S, Zwecker CL, Fleiss JL. Some statistical methods useful in circulation research. Circ Res 1980; 47:1-9.

11 Van der Tweel I, Herbschleb JN, Borst C, Meijler FL. Deterministic model of the canine atrioventricular node as a periodically perturbed, biological oscillator. $J$ App a periodically perturbed
Cardiol 1986;1:157-73.

12 Wittkampf FHM, De Jongste MJL, Lie HI, Meijler FL. Effect of right ventricular pacing on ventricular rhythm during

13 Meijler FL, Fisch C. Does the atrioventricular node conduct? Br Heart $J$ 1989;61:309-15.

14 Borst C, Meijler FL. Baroreflex modulation of ventricular rhythm in atrial fibrillation. Eur Heart $J 1984 ; 5: 870-5$.

15 Meijler FL, Kroneman J, Van der Tweel I, Herbschleb JN, Heethaar RM, Borst C. Nonrandom ventricular rhythm in horses with atrial fibrillation and its significance for patients. J Am Coll Cardiol 1984;4:316-23.

16 Kirsh JA, Sahakian AV, Baerman JM, Swiryn S. Ventricular response to atrial fibrillation: role of atrioventricular conduction pathways. J Am Coll Cardiol 1988;12. 1265-72.

17 Dreifus LS, Mazgalev T. "Atrial paralysis": does it explain the irregular ventricular rate during atrial fibrillation. $J A m$ the irregular ventricular rate

18 Meijler FL An "account" of digitalis and atrial fibrillation. $J$ Am Coll Cardiol 1985;5:60A-8A

19 Hoffmann BF, Bigger JT. Digitalis and allied cardiac glycosides. In: Gilman AG, Goodman LS, Rall TW, Murad F, eds. The pharmacological basis of therapeutics. New York: Macmillan, 1980:729-60.

20 Langendorf R, Pick A, Katz LN. Ventricular response in atrial fibrillation: role of concealed conduction in the AV junction. Circulation 1965;32:69-75

21 Härtel G, Kyllonen K, Merikallio E, Ojala K, Manninen V, Reissel P. Human serum and myocardium digoxin. Clin Pharmacol Ther 1976;19:153-7.

22 Gullner HG, Stinson EB, Harrison DC, Kalman SM. Correlation of serum concentrations with heart concentrations of digoxin in human subjects. Circulation 1974; 50:653-5.

23 Lloyd BL, Taylor RR. Influence of myocardial mechanical activity and coronary blood flow on myocardial digoxin uptake Cardiovasc Res 1976;10:487-93.

4 Lloyd BL, Taylor RR. The effect of heart rate on myocardia oubain uptake and on the susceptibility to oubain car diotoxicity in the dog. Clin Exp Pharmacol Physio 1978;5:171-9.

25 Chamberlain DA, White RJ, Howard MR, Smith TW Plasma digoxin concentrations in patients with atrial fibrillation. $B M J$ 1970;iii:429-32.

26 Halkin H, Kleiner A, Almog S, Millman P, Tirosh M. Value of serum digoxin concentration measurement in the control of digoxin therapy in atrial fibrillation. Isr $J$ Med Sci 1979;15:490-3.

27 Fogelmann AM, La Mont JT, Finkelstein S, Rado E. Fallibility of plasma digoxin differentiating toxic from non-toxin patients. Lancet $1971 ; \mathbf{i i}: 727-9$.

28 Jogestrand T, Nordlander R. Serum digoxin determination in outpatients-need for standardization. Br J Clin Pharmacol 1983;15:55-8.

29 Laursen S $\emptyset$, Pedersen KE, Klitgaard NA. Influence of physical activity on plasma digoxin in hospitalised 1987:34:115-7.

30 Redfors A. Digoxin dosage and ventricular rate at rest and exercise in patients with atrial fibrillation. Acta Med Scand 1971;190:321-33.

31 Smith TW. Digitalis. Mechanisms of action and clinical use $N$ Engl $J$ Med 1988;318:358-65.

32 Lewis R, Lakkani M, Moreland TA, McDewitt DG. A comparison of verapamil and digoxin in the treatment of atrial fibrillation. Eur Heart $J$ 1987;8:148-53.

33 Roth A, Harrison E, Mitani G, et al. Efficacy and safety of medium-and high dose diltiazem alone and in combination with digoxin for control of heart rate at rest and during exercise in patients with atrial fibrillation. Circulation 1986;73:316-24.

34 Steinberg JS, Katz'RJ, Bren GB, Buff LA, Varghese PJ. Efficacy of oral diltiazem to control ventricular response in chronic atrial fibrillation at rest and during exercise. $J \mathrm{Am}$ Coll Cardiol 1987;9:405-11.

35 Lewis R, Laing E, Moreland TA, Service E, McDewitt DG. A comparison of digoxin, diltiazem and their combination A comparison of digoxin, diltiazem and their combination in the treatment

36 DiBianco R, Morganroth J, Freitag JA, et al. Effects of nadolol on the spontaneous and exercise provoked hear rate of patients with chronic atrial fibrillation receivin stable doses of digoxin. Am Heart $J$ 1984;108:1121-7.

37 Lang R, Klein HO, Weiss E, et al. Superiority of ora verapamil therapy to digoxin in the treatment of chronic atrial fibrillation. Chest 1983;83:491-9.

38 Brown RW, Goble AJ. Effect of propranolol on exercise tolerance of patients with atrial fibrillation. $B M$ 1969;ii:279-80

39 David D, DiSegni E, Klein HO, Kaplinsky E. Inefficacy of digitalis in the control of heart rate in patients with chronic digitrich gic blocking agent. Am J Cardiol 1979;44:1379-82. 